\title{
ON THE DISTRIBUTION OF VALUES OF MEROMORPHIC FUNCTIONS
}

\author{
BY \\ FRED GROSS
}

1. Introduction. It is well known that the growth of a meromorphic function is closely related to the distribution of its poles and zeros. One would, therefore, expect that two meromorphic functions $f(z)$ and $g(z)$ would have the same growth if for certain appropriate sets $S_{i}, f \in S_{i}$ if and only if $g \in S_{i}, i=1,2, \ldots, k ; k$ some positive integer. It was proven by Nevanlinna (see Hayman [1]), for example, that for any two meromorphic functions $f_{1}(z)$ and $f_{2}(z)$, if the zeros of $f_{1}(z)-a$ are the same as the zeros of $f_{2}(z)-a$ for five distinct values of $a$, then $f_{1}(z) \equiv f_{2}(z)$ or $f_{1}, f_{2}$ are both constant. In this paper we shall consider pairs of meromorphic functions $f, g$ such that $f \in S$ iff $g \in S$ for certain sets $S$ of complex numbers and see how these functions $f$ and $g$ must be related.

2. Preliminaries. We begin with some of the theorems needed in the proof of our main results.

It is assumed that the reader is familiar with the definitions and basic properties of the quantities $T(r, f), N(r, f), \log ^{+}$, etc.

THEOREM 1. Let $\phi_{1}(z), \phi_{2}(z), \ldots, \phi_{n}(z)$ be $n$ entire functions with $\phi_{i}-\phi_{j}$ nonconstant for $i \neq j$; and let $g_{1}(z), g_{2}(z), \ldots, g_{n}(z)$ be $n$ meromorphic functions of finite order such that the order of $g_{1}(z)<\rho, i=1,2, \ldots, n$, where $\rho$ is the minimum of the orders of the functions $\exp \left(\phi_{s}-\phi_{t}\right)(s \neq t) s=1, \ldots, n ; t=1, \ldots, n$.

If $\sum_{1}^{n} g_{i}(z) \exp \left(\phi_{i}(z)\right)=0$, then $g_{1}=g_{2}=\cdots=g_{n}=0$.

In order to state Theorem 2 we need

Definition 1. To each function $\lambda(r)$, positive, continuous and nondecreasing on $0 \leqq r<R$, where $R \leqq+\infty$, we associate the class $\Lambda$ of functions $f$ satisfying

(i) $f$ is meromorphic in $|z|<R$,

(ii) $T(r, f)=O(\lambda(r))$ as $r \rightarrow R$.

It is easily verified that $\Lambda$ is a field, and we call any such field a $\Lambda$-field.

THEOREM 2. Each $\Lambda$-field is algebraically closed in the field of all functions meromorphic in $|z|<R$.

Theorem 1 follows from a more general result of Nevanlinna [7]. Theorem 2 is due to Rubel and Hellerstein [3].

Received by the editors March 20, 1965 and, in revised form, January 6, 1967. 
We shall also need

LeMma 1. Let $F_{i}\left(x_{1}, \ldots, x_{n}, y_{i}\right)=0 ; i=1, \ldots, n+1$, where $F_{i} \not \equiv 0$ are polynomials in $n+1$ variables. There exists a polynomial, $P \not \equiv 0$ such that $P\left(y_{1}, \ldots, y_{n+1}\right)=0$.

This can easily be proved by induction.

LEMMA 2. The characteristic function $T(r, f)$ is continuous in $r$ for every meromorphic function $f(z)$.

Proof. This follows from an argument which can be found in Titchmarsh [6, p. 127 , line 5].

LEMMA 3. $T(r, f)$ is an increasing convex function of $\log r$ for any meromorphic function $f(z)$.

Proof. See Titchmarsh [6, p. 284d].

DEFINITION 2. Given any two meromorphic functions $f(z)$ and $g(z)$ we shall say that the growth of $f(z)$ is greater than the growth of $g(z)$, denoted by $G(f)>G(g)$, if and only if $T(r, g)=O(T(r, f))$.

Definition 3. Two entire functions $f(z)$ and $g(z)$ are said to have the same growth, denoted by $G(f)=G(g)$, if and only if $G(f)>G(g)$ and $G(g)>G(f)$.

Note. According to this definition two functions of a given order and different types may be of the same growth.

\section{Two functions attaining certain values at same points.}

Definition 4. For any set $S$ and any function $g$ let

$$
E g(S)=\bigcup_{a \in S}\{\xi ; g(\xi)-a=0\},
$$

where any $\xi$ which is a zero of multiplicity $m$ is included in $\operatorname{Eg}(S) m$ times.

THEOREM 3. Let $S_{i}, i=1,2,3$ be distinct finite sets of complex numbers such that no one of them is equal to the union of the other two and let $T_{i}$, be any finite sets of complex numbers having the same number of elements as $S_{i} ; i=1,2,3$. Let $f(z)$ and $g(z)$ be two meromorphic functions such that $E_{f}\left(S_{i}\right)=E_{g}\left(T_{i}\right)$ and $E_{1 / f}\{0\}=E_{1 / g}\{0\}$ for $i=1,2,3$. Then $f(z)$ and $g(z)$ are algebraically dependent.

(NotE. In this and in the following theorems $S_{i}$ and $T_{i}$ need not have the same number of elements if $f$ and $g$ are assumed to be entire.)

Proof. Let $z_{i j}, j=1, \ldots, n_{i}$ be the elements of $S_{i}$ and $z_{i j}^{\prime}, j=1, \ldots, n_{i}$ be those of $T_{i}$ for $i=1,2,3$.

Let

and

$$
p_{i}(w)=\prod_{j=1}^{n_{i}}\left(w-z_{i j}\right)
$$

$$
q_{i}(w)=\prod_{j=1}^{n_{i}}\left(w-z_{i j}^{\prime}\right)
$$


We have

$$
\frac{p_{i}(f(z))}{q_{i}(g(z))}=\exp \left(\phi_{i}(z)\right)
$$

where $\phi_{i}(z)$ is entire for $i=1,2,3$.

It follows from Lemma 1 that $\exp \left(\phi_{1}(z)\right)$, $\exp \left(\phi_{2}(z)\right)$ and $\exp \left(\phi_{3}(z)\right)$ are algebraically dependent. Hence, there exists a relation of the form

$$
\sum_{n, m, t} \lambda_{n m t} \exp \left(n \phi_{1}+m \phi_{2}+t \phi_{3}\right)=0,
$$

where $\lambda_{n m t}$ are complex numbers and not all constant.

Applying Theorem 1 to (1) we find that there exist integers $a, b$ and $c$ such that $a \phi_{1}(z)+b \phi_{2}(z)+c \phi_{3}(z)=k$, where $k$ is some constant.

Hence

$$
\left(p_{1}(f) / q_{1}(g)\right)^{a} \cdot\left(p_{2}(f) / q_{2}(g)\right)^{b} \cdot\left(p_{3}(f) / q_{3}(g)\right)^{c}=\text { constant. }
$$

Thus either $f(z)$ and $g(z)$ are algebraically dependent or $\left(p_{1}(w)\right)^{a}\left(p_{2}(w)\right)^{b}\left(p_{3}(w)\right)^{c}$ is equal to some constant $k^{\prime}$. The latter statement, however, implies that

$$
\left(p_{1}(w)\right)^{a}\left(p_{2}(w)\right)^{b}=\left(p_{3}(w)\right)^{-c} \cdot k^{\prime}
$$

and consequently that

$$
S_{1} \cup S_{2}=S_{3}
$$

contrary to our hypothesis and our theorem follows.

Corollary to Theorem 3. Let $S_{i}, T_{i}, f$ and $g, i=1,2,3$ be as in Theorem 3 , then $f(z)$ and $g(z)$ have the same growth.

Proof. Since $T(r, f)$ is positive, continuous, and nondecreasing (Lemmas 2 and 3) for any meromorphic function $f$, we can apply Theorem 2 to the algebraically dependent functions $f$ and $g$ and we get $T(r, g)=O(T(r, f))$ and $T(r, f)=O(T(r, g))$. Hence $G(f)=G(g)$.

In what follows $f^{(0)}(z)$ denotes $f(z)$.

THEOREM 4. Let $S_{i}$ and $T_{i}$ be as in Theorem 3. Let $f(z)$ and $g(z)$ be any two meromorphic functions of finite order. If for some nth derivative $f^{(n)}(z)$ of $f(z)$ and some mth derivative $g^{(m)}(z)$ of $g(z), E_{f^{(n)}}\left(S_{i}\right)=E_{g^{(m)}}\left(T_{i}\right)$ and $E_{1 / f^{(n)}}(\{0\})=E_{1 / g^{(m)}}(\{0\})$, $i=1,2,3$, then $f(z)$ and $g(z)$ have the same order.

Proof. Let the order of $f^{(n)}$ be $\rho_{1}$ and that of $g^{(m)}$ be $\rho_{2}$. Since

and

$$
\rho_{1}=\limsup _{r \rightarrow \infty} \frac{\log T\left(r, f^{(n)}\right)}{\log r}, \quad \rho_{2}=\limsup _{r \rightarrow \infty} \frac{\log T\left(r, g^{(m)}\right)}{\log r}
$$

$$
G\left(f^{(n)}\right)=G\left(g^{(m)}\right)
$$

it follows that $\rho_{1}=\rho_{2}$. Furthermore, it is known (see Hayman [1, p. 104]) that the order of the derivative $f^{\prime}(z)$ is the same as the order of $f(z)$ and our theorem follows. 
THEOREM 5. Let $S_{i}$ and $T_{i}, i=1,2$, be any two distinct finite sets of complex numbers where $S_{i}$ and $T_{i}$ have the same number of elements. Let $f(z)$ and $g(z)$ be a pair of meromorphic functions of order less than 2 . If for some integers $m \geqq 0$ and $n \geqq 0, E_{f^{(n)}}\left(S_{i}\right)=E_{g^{(m)}}\left(T_{i}\right)$ and $E_{1 / f^{(n)}}(\{0\})=E_{1 / g^{(m)}}(\{0\})$ for $i=1,2$, then $f(z)$ and $g(z)$ have the same order.

Proof. As in the proof of Theorem 3, let $p_{i}(w)$ and $q_{i}(w)$ correspond to $S_{i}$ and $T_{i}$, respectively, for $i=1,2$.

It suffices to assume that the hypotheses hold for $f$ and $g$ (see Hayman [1, p. 104j).

We have

$$
p_{1}(f(z)) / q_{1}(g(z))=\exp \left(\phi_{1}(z)\right) \quad \text { and } \quad p_{2}(f(z)) / q_{2}(g(z))=\exp \left(\phi_{2}(z)\right),
$$

where $\phi_{1}(z)$ and $\phi_{2}(z)$ are polynomials.

It follows from Lemma 1 that $g(z), \exp \left(\phi_{1}(z)\right)$ and $\exp \left(\phi_{2}(z)\right)$ as well as $f(z)$, $\exp \left(\phi_{1}(z)\right)$ and $\exp \left(\phi_{2}(z)\right)$ are algebraically dependent.

Assume that our theorem is false and that $f(z)$ is of greater order than $g(z)$. One can easily verify that for any polynomial $p(w)$ and any meromorphic function $f(z)$ of finite order, the order of $f(z)=$ order of $p(f(z))$. It follows that the orders of $\exp \left(\phi_{1}(z)\right), \exp \left(\phi_{2}(z)\right)$ and $f(z)$ are all equal. Hence, $f(z)$ must be of integral order. Since by hypothesis the order of $f(z)$ is less than 2 , it can be at most of order 1 . Thus, $g(z)$ must be of order less than 1. Since $g(z)$, exp $\left(\phi_{1}(z)\right)$ and $\exp \left(\phi_{2}(z)\right)$ are algebraically dependent we must have a relation of the form

$$
\sum_{n, m, t} \lambda_{n m t} g^{n} \exp \left(m \phi_{1}+t \phi_{2}\right)=0 .
$$

One can now apply Theorem 1 and arrive at $j \phi_{1}(z)+k \phi_{2}(z)=$ constant, for some integers $j$ and $k$ with $j$ or $k$ different from zero. Hence we get

$$
p_{1}(f(z))^{j} p_{2}(f(z))^{k}=q_{1}(g(z))^{j} q_{2}(g(z))^{k},
$$

where $q_{i}(w)$ are polynomials. Hence, either $f(z)$ and $g(z)$ are algebraically dependent and hence of the same order or $p_{1}(w)^{j} p_{2}(w)^{k}=$ constant, in which case $S_{1}=S_{2}$. In any case we get a contradiction. Thus, $f(z)$ and $g(z)$ must be of the same order.

4. A specific case. In this section we illustrate how additional information about the sets $S_{i}, i=1,2,3$ may enable one to completely determine $f(z)$ and $g(z)$.

THEOREM 6. Let $f$ and $g$ be nonconstant entire functions such that $f \in S_{i}$ if and only if $g \in S_{i}$ for $i=1,2$, and 3 with the same multiplicities, where $S_{1}=\{1\}, S_{2}=\{-1\}$ and $S_{3}=\left\{a_{3}, a_{4}\right\}, S_{i} \cdot S_{j}=\varnothing$ for $i \neq j$. Then $f$ and $g$ must satisfy one of the following relations:

$$
f=g, \quad f g=1 \quad \text { or } \quad(f-1)(g-1)=4 .
$$

Since the proof of this theorem is quite lengthy, we shall give only an outline of the proof. 
SKeTCH OF ProOF. From the hypotheses of the theorem, one obtains

$$
\begin{aligned}
& f=\exp \left(\phi_{1}\right) g+\left(1-\exp \left(\phi_{1}\right)\right), \\
& f=\exp \left(\phi_{2}\right) g+\exp \left(\phi_{2}\right)-1
\end{aligned}
$$

and

$$
f^{2}-\left(a_{3}+a_{4}\right) f+a_{3} a_{4}=\exp \left(\phi_{3}\right)\left(g^{2}-\left(a_{3}+a_{4}\right) g+a_{3} a_{4}\right),
$$

where $\phi_{i}$ are entire functions. Eliminating $f$ and $g$ above we get

$$
\begin{aligned}
& -4 \exp \left(3 \phi_{1}\right)+\left(3-\left(a_{3}+a_{4}\right)-a_{3} a_{4}\right) \exp \left(2 \phi_{1}\right)+\left(1-\left(a_{3}+a_{4}\right)+a_{3} a_{4}\right) \exp \left(2 \phi_{2}\right) \\
& +4 \exp \left(3 \phi_{1}+\phi_{2}\right)+\left(-4+2\left(a_{3}+a_{4}\right)\right) \exp \left(2 \phi_{1}+\phi_{2}\right) \\
& +\left(-4+2\left(a_{3}+a_{4}\right)\right) \exp \left(\phi_{1}+2 \phi_{2}\right) \\
& +\left(4-2\left(a_{3}+a_{4}\right)\right) \exp \left(\phi_{1}+\phi_{2}\right)+\left(1-\left(a_{3}+a_{4}\right)+a_{3} a_{4}\right) \exp \left(\phi_{3}+2 \phi_{1}\right) \\
& +\left(2\left(a_{3}+a_{4}\right)\right) \exp \left(\phi_{3}+\phi_{1}\right)+\left(3-a_{3} a_{4}-\left(a_{3}+a_{4}\right)\right) \exp \left(\phi_{3}+2 \phi_{2}\right) \\
& +\left(-4+2\left(a_{3}+a_{4}\right)\right) \exp \left(\phi_{3}+\phi_{2}\right)-\left(2\left(a_{3}+a_{4}\right)\right) \exp \left(\phi_{1}+\phi_{2}+\phi_{3}\right)=0 .
\end{aligned}
$$

In the following, two terms will be said to cancel if their exponents differ by a constant. We now apply Theorem 1 to (8). The first seven terms of the left side of equation (8) do not involve $\exp \left(\phi_{3}\right)$, while the last five do. If any two nonvanishing terms in either of these two groups cancel with each other we get a relation of the form $\exp \left(\phi_{1}\right)=k \exp \left(c \phi_{2}\right)$ ( $c$ rational). If no cancellation occurs in the first group, then two of these first seven terms must vanish identically.

Thus we have the following identity

$$
\begin{aligned}
-4 \exp \left(3 \phi_{1}\right) & +\left(1-a_{3} a_{4}\right) \exp \left(2 \phi_{1}\right)+\left(a_{3} a_{4}-1\right) \exp \left(2 \phi_{2}\right)+4 \exp \left(3 \phi_{1}+\phi_{2}\right) \\
& +\left(a_{3} a_{4}-1\right) \exp \left(\phi_{3}+2 \phi_{1}\right)+4 \exp \left(\phi_{3}+\phi_{1}\right)+\left(1-a_{3} a_{4}\right) \exp \left(\phi_{3}+2 \phi_{2}\right) \\
& -4 \exp \left(\phi_{1}+\phi_{2}+\phi_{3}\right)=0
\end{aligned}
$$

and since $a_{3} \neq 1$ by hypothesis, none of the coefficients are zero.

Now $\exp \left(3 \phi_{1}\right)$ can cancel with one of 4 terms and we have

(a) $3 \phi_{1}=\phi_{3}+2 \phi_{1}$ or $\phi_{3}=\phi_{1}$,

(b) $3 \phi_{1}=\phi_{3}+\phi_{1}$ or $\phi_{3}=2 \phi_{1}$,

(c) $3 \phi_{1}=\phi_{3}+2 \phi_{2}$ or $\phi_{3}=3 \phi_{1}-2 \phi_{2}$,

(d) $3 \phi_{1}=\phi_{1}+\phi_{2}+\phi_{3}$ or $\phi_{3}=2 \phi_{1}-\phi_{2}$.

(For convenience the constants of these equalities are omitted.)

Careful analysis of the various cases which arise leads to the conclusion that either $f$ and $g$ satisfy one of the equations (4) or

$$
\exp \left(\phi_{1}\right)=k \exp \left(c \phi_{2}\right)
$$

where $c$ is one of the numbers $2,-1,2 / 3,1$ and $1 / 2$.

This takes care of the situation when no cancellation occurs in the first group of seven or in the second group of five terms. 
When cancellation occurs within the first group a somewhat similar analysis leads to the possible relations $\exp \left(\phi_{1}\right)=k \exp \left(c \phi_{2}\right)$ where $c$ is one of the numbers $2 / 3,1 / 2,2,1$ and -1 .

Further investigation shows that $c$ cannot equal 2/3. The remaining possibilities lead to one of the equations (4).

Corollary. Let $S_{1}=\{1\}, S_{2}=\{-1\}, S_{3}=\left\{a_{3}, a_{4}\right\}, S_{4}=\left\{b_{1}, \ldots, b_{n}\right\}$ be such that $S_{i} \cdot S_{j}=\varnothing$ for $i \neq j$ where $n$ is an odd integer. If $f \in S_{i}$ if and only if $g \in S_{i}$ with the same multiplicities for $i=1,2,3$ and 4 and if $b_{j} \neq 0$ or 3 for $j=1,2, \ldots, n$ then $f=g$.

Proof. Assume that $f \neq g$. Looking at the remaining possibilities we find that if some $b_{j}$ is not attained then $b_{j}$ must be 0 and if all are attained then some $b_{j}$ must be 3 .

This result can be generalized to functions of several complex variables. Once the following definition is introduced the proof remains the same.

Let $f$ and $g$ be entire functions of two (or more) complex variables. If $f$ vanishes at the origin, then by virtue of the Weierstrass preparation theorem $f(z, w)=$ $\Pi_{1}(z, w) \Omega_{1}(z, w)$ in a neighborhood $U$ of the origin, where $\Pi_{1}$ is a polynomial in $w$ with functions of $z$, analytic on $U$, as coefficients and where $\Omega_{1}$ is nonvanishing and analytic on $U$.

Definition 5. Let $f(z, w)$ and $g(z, w)$ be two entire functions such that $f=0$ if and only if $g=0$. A common zero $\left(\xi_{1}, \xi_{2}\right)$ of $f$ and $g$ is said to be a zero of the same multiplicity with respect to both functions $f$ and $g$ if and only if $\Pi_{1}=\Pi_{2}$, where

$$
f\left(z+\xi_{1}, w+\xi_{2}\right)=\Pi_{1}\left(z+\xi_{1}, w+\xi_{2}\right) \cdot \Omega\left(z+\xi_{1}, w+\xi_{2}\right)
$$

and

$$
g\left(z+\xi_{1}, w+\xi_{2}\right)=\Pi_{2}\left(z+\xi_{1}, w+\xi_{2}\right) \Omega_{2}\left(z+\xi_{1}, w+\xi_{2}\right) .
$$

An equivalent definition to Definition 5 is

Definition 6. Two entire functions $f$ and $g$ have the same zeros with the same multiplicities if and only if $f / g=\exp (\phi)$, where $\phi$ is entire.

5. Images and pre-images of certain sets. In this section we give some applications of the results of $\S 3$. Though these applications, as we shall see, yield much weaker results about reduced sets (defined below) than the direct application of Nevanlinna's second fundamental theorem, nevertheless the methods used and the intermediate theorems are interesting in themselves and hence have been included in this paper. Certain generalizations of reduced sets are also discussed.

Definition 7. A set $S$ of complex numbers is a simply attained set of an entire function $f(z)$ iff, for every $s \in S, f-s$ has only simple zeros.

Definition 8. Let $f(z)$ be meromorphic and $S$ be a set of complex numbers such that $f(S)=S$. Then $S$ is said to be a reduced set of $f(z)$ iff it is a simply attained set of $f(z)$ and $f(z) \in S$ implies that $z \in S$.

THEOREM 7. Let $f(z)$ be a transcendental entire function. Let $S_{1}, S_{2}$, and $S_{3}$ be 
finite sets of complex numbers such that no one is the union of the other two. If $S_{1}, S_{2}$, and $S_{3}$ are simply attained sets of $f(z)$, then $f^{-1}\left(S_{1}\right), f^{-1}\left(S_{2}\right)$ and $f^{-1}\left(S_{3}\right)$ cannot all be reduced sets of any transcendental entire function $g(z)$.

Proof. Assume that the theorem is false. It then follows that $E_{f}\left(S_{i}\right)=E_{f(g)}\left(S_{i}\right)$ for $i=1,2,3$. Hence by the corollary to Theorem 3 it follows that $f(z)$ and $f(g(z))$ must have the same growth. It is well known (Hayman [1]), however, that $T(r, f(g)) / T(r, f) \rightarrow \infty$ as $r \rightarrow \infty$, so that the growth of $f(g(z))$ must be greater than the growth of $f$.

THEOREM 8. Let $f(z)$ and $g(z)$ be two nonconstant entire functions with a common Picard exceptional point a. If $\mathrm{S}, \not \equiv\{a\}$, is any finite set of complex numbers and $E_{f}(S)=E_{g}(S)$, then $f(z)=e^{\phi(z)}+a$ and $g(z)$ is either of the form $c e^{\phi(z)}+a$ with $c^{n}=1$ for some integer $n$, or of the form $k e^{-\phi(z)}+a$, where $k$ is a constant and $\phi(z)$ is an entire function.

Proof. We may assume without any loss of generality that $a=0$, so that $f(z)=e^{\phi(z)}$ and $g(z)=e^{\gamma(z)} ; \phi(z)$ and $\gamma(z)$ entire. As in Theorem 5 we find a polynomial $p(w)=w^{n}+\lambda_{1} w^{n-1}+\cdots+c$ (we may assume $c \neq 0$ ) such that

$$
p\left(e^{\phi(z)}\right) / p\left(e^{\gamma(z)}\right)=e^{\eta(z)} ; \quad \eta(z) \text { some entire function. }
$$

Thus we get

$$
e^{n \phi}+\lambda_{1} e^{(n-1) \phi}+\cdots+c=e^{n \gamma+n}+\lambda_{1} e^{(n-1) \gamma+\eta}+\cdots+c e^{\eta} .
$$

By virtue of Theorem 1 we conclude that $n \phi=j \gamma+\eta+c_{1}$ and $t \gamma+\eta=c_{2}$, where $c_{1}$ and $c_{2}$ are constants. Hence $\phi=c \gamma+c_{3}$, where $c$ is a rational number and $c_{3}$ is a constant.

Thus $e^{\phi}=\exp \left(c \gamma+c_{3}\right)$. By our hypotheses, however, $\exp \left(\gamma\left(x_{0}\right)\right) \in S$ implies that $\exp \left(\phi\left(x_{0}\right)\right)=\exp \left(c \gamma\left(x_{0}\right)+c_{3}\right)$ is also in $S$. Thus

$$
\exp \left(c^{n} \gamma\left(x_{0}\right)+c_{3}\left(1+c+c^{2}+\cdots+c^{n-1}\right)\right) \in S
$$

for all $n$. If $|c| \neq 1$ then we may assume that $|c|<1$ and we get

$$
\begin{aligned}
c^{n} \gamma\left(x_{0}\right)+c_{3}\left(1+c+c^{2}+\cdots+c^{n-1}\right) & =c^{n} \gamma\left(x_{0}\right)+c_{3}\left(\left(c^{n}-1\right) /(c-1)\right) \\
& =c^{n}\left(\gamma\left(x_{0}\right)+c_{3} /(c-1)\right)-c_{3} /(c-1) .
\end{aligned}
$$

If $\gamma\left(x_{0}\right)=-c_{3} /(c-1)$, then we may aplace $c_{3}$ by $c_{3}+2 \pi i$, so that we may assume $\gamma\left(x_{0}\right) \neq-c_{3} /(c-1)$. Hence we get an infinite sequence of complex numbers approaching a constant as $n$ approaches infinity. Consequently the numbers

$$
\exp \left(c^{n} \gamma\left(x_{0}\right)+c_{3}\left(1+c+c^{2}+\cdots+c^{n-1}\right)\right)
$$

are distinct for infinitely many $n$ which is impossible since $S$ is finite.

It follows that $|c|=1$ and, since $c$ is rational, $c= \pm 1$.

When $c=1$, we get $f=k g$ where $k$ is a constant. When $g(x) \in S$, so is $f(x)=k g(x)$ and hence $k^{n} g(x) \in S$ for every $n$. Thus $k$ must be a root of unity. Our proof is complete. 
One can easily find a number of sets $S$ for which $f(z)$ and $g(z)$, with $c= \pm 1$, have the desired property.

As an application of Theorem 8 we give an alternate proof of the well-known fact:

THEOREM 9. Let $f(z)$ be any entire function and let I denote the set of all rational integers. If $E_{f}(I)=I$, then $f(z)$ must be of the form $A z+B$, where $A$ is \pm 1 and $B$ is an integer.

Proof. $e^{2 \pi i z}$ is never zero, is equal to 1 if and only if $z \in I$, and all the roots of $e^{2 \pi i z}=1$ are simple roots. Let $f(z)$ be an entire function such that $E_{f}(I)=I$. Then $e^{2 \pi i f(z)}$ is equal to 1 if and only if $f(z) \in I$ and hence if and only if $z \in I$. Thus $e^{2 \pi i z}$ and $e^{2 \pi i f(z)}$ satisfy the hypotheses of Theorem 8 and hence $e^{2 \pi i f(z)}$ must have either the form $c e^{-2 \pi i z}$ or $c e^{2 \pi i z}$, so that $f(z)$ must be of the form $\pm z+B$. Since this has integral values at the integers, $B$ must be an integer and our theorem follows.

We now prove a generalization of Theorem 9. The author is indebted to E. G. Straus for suggesting the proof of the following theorem.

THEOREM 10. Let $F(z)=c_{1} \exp \left(a_{1} z\right)+\cdots+c_{n} \exp \left(a_{n} z\right)$ with $a_{i} \neq a_{j}(n \geqq 2)$ for $i \neq j$, where $c_{i}$ are constant for $i=1,2, \ldots, n$. Let $Z=\left\{z_{1}, z_{2}, \ldots\right\}$ be the zeros of $F(z)$. If all the zeros of $F(z)$ are simple zeros and $E_{f}(Z)=E_{g}(Z)$ for any two entire functions $f(z)$ and $g(z)$ then $f(z)=A g(z)+B$ where $A$ is a certain root of unity and $B$ an appropriate constant.

Proof. From our hypotheses it follows that $F(f(z)) / F(g(z))$ is entire and has no zeros so that we have

$$
F(f(z))=F(g(z)) e^{\phi(z)}
$$

where $\phi(z)$ is entire. By Theorem 1,

$$
a_{i} f(z)=a_{i} g(z)+\phi(z)+b_{i} \quad \text { for } i=1, \ldots, n .
$$

Since $n \geqq 2$ we get at least two such equations and it follows that $f(z)=A g(z)+B$ and $\phi(z)=C g(z)+D$ for some constants $A, B, C$, and $D$. We may assume $A \neq 1$. Substituting we get

$$
\sum_{i=1}^{n} C_{i} \exp \left(a_{i}(A g+B)\right)=\sum_{i=1}^{n} C_{i} \exp \left(\left(a_{i}+C\right) g+D\right) .
$$

We now introduce vector notation using inner product multiplication. Let $a=\left\{a_{1}, \ldots, a_{n}\right\}$ and $1^{*}=(1,1, \ldots, 1)$. We have $a A=a+C \cdot 1^{*}$,

$$
a A^{2}=a A+C A \cdot 1^{*}=a+C \cdot 1^{*}+C A \cdot 1^{*}=a+(1+A) C \cdot 1^{*},
$$

and in general

$$
a A^{n}=a+\left(1+A+A^{2}+\cdots+A^{n-1}\right) C \cdot 1^{*},
$$

so that

$$
a=a \mid A^{n}+1^{*} \cdot\left(\left(A^{n}-1\right) /(A-1) A^{n}\right) C \rightarrow 0+(C /(A-1)) \cdot 1^{*} \quad \text { as } n \rightarrow \infty,
$$


unless $|A|=1$. Here 0 denotes the 0 vector. Since this is impossible by our hypothesis, we must have $|A|=1$.

Assume that $A$ is not a root of unity. Then the expression for $a$, namely

$$
a A^{-n}+\frac{1-A^{n}}{(1-A) A^{n}} C \cdot 1^{*}=A^{-n}\left(a+\frac{C}{1-A} \cdot 1^{*}\right)-\frac{C}{1-A} \cdot 1^{*}
$$

attains infinitely many values unless

$$
a=-(C /(1-A)) \cdot 1^{*} \quad \text { or } \quad a_{i}=-C /(1-A) \quad \text { for } i=1, \ldots, n,
$$

which is contrary to our hypothesis in any case. Thus $A$ must be some root of unity.

We note that any $F(z)$ with $n=2$ can have only simple zeros. Hence applying Theorem 10 to $\sin \pi z$ one can easily derive Theorem 9 .

We now proceed to develop an extension of Nevanlinna's second fundamental theorem and to apply the result to a number of problems involving reduced sets.

Consider the polynomials $\eta(w)=\prod_{i=1}^{k}\left(w-a_{i}\right), a_{i}$ distinct. Let $A=\max _{i \leq k}\left\{\left|a_{i}\right|, 1\right\}$. The polynomials

and

$$
P(x)=\eta(x) \sum_{i=1}^{k} \frac{1}{\left(\eta^{\prime}\left(a_{i}\right)\right)^{2}\left(x-a_{i}\right)}
$$

$$
Q(x)=\sum_{i=1}^{k} \frac{\eta^{\prime}\left(a_{i}\right)-\eta^{\prime}(x)}{\left(x-a_{i}\right)\left(\eta^{\prime}\left(a_{i}\right)\right)^{2}}
$$

satisfy $P \eta^{\prime}+Q \eta=1$. When $|x| \leqq 2 A$ one can show after some calculations that

$$
\max (|P(x)|,|Q(x)|) \leqq k(3 A)^{k-1} / \delta(k)^{2(k-1)}=k_{1},
$$

where $\delta(k)$ is the minimum of the distances between the points $a_{i}$.

Let $f$ be a meromorphic function such that $f(0) \neq a_{i}, 0, \infty$.

Let $S(k)=\{r ; r>A\}$.

When $x>2 A|\eta(x)| \geqq|x / 2|^{k}$, so that if $|\eta(x)| \leqq 1$, then $|x| \leqq 2 A$. Thus when $|\eta(f)| \leqq 1$ one easily verifies that

$$
\begin{aligned}
m\left(r, \frac{1}{(f)}\right) \leqq & 2 \log ^{+} k_{1}+\log 2+m\left(r, \frac{\eta^{\prime}(f) f^{\prime}}{\eta(f)}\right) \\
& +m\left(r, \frac{f^{\prime}}{f}\right)+N\left(r, f^{\prime}\right)-N\left(r, \frac{1}{f^{\prime}}\right)+m(r, f)-\log \left|C_{\lambda}^{\prime}\right|,
\end{aligned}
$$

where $f^{\prime}(z)=C_{\lambda}^{\prime} z^{\lambda}+\cdots ; C_{\lambda}^{\prime} \neq 0$.

Thus

\section{LEMMA 4.}

$$
\begin{aligned}
m\left(r, \frac{1}{\eta(f)}\right) \leqq & 2 \log ^{+} k_{1}+\log 2+m\left(r, \frac{\eta^{\prime}(f) f^{\prime}}{\eta(f)}\right) \\
& +m\left(r, \frac{f^{\prime}}{f}\right)+N\left(r, f^{\prime}\right)-N\left(r, \frac{1}{f^{\prime}}\right)+m(r, f)-\log \left|C_{\lambda}^{\prime}\right|
\end{aligned}
$$

for all $r$ in $S(k)$. 
LEMMA 5.

$$
\begin{aligned}
m\left(r, \frac{f^{\prime}}{f}\right)< & 4 \log ^{+} T(R, f)+4 \log ^{+} \log ^{+} \frac{1}{|f(0)|} \\
& +5 \log ^{+} R+6 \log ^{+} \frac{1}{R-r}+\log ^{+} \frac{1}{r}+14
\end{aligned}
$$

$R>r$ for all $r$.

Proof. Hayman [1].

LEMMA 6. $m\left(r, f^{\prime} \mid f\right)=O(\log r T(r, f))$ for all $r$ outside a set, $E_{0}$, of finite measure.

Proof. Hayman [1].

Using Lemma 5, some standard Nevanlinna arguments and the fact that

$$
|\eta(f(0))|=\left|\prod_{i=1}^{k}\right| f(0)-a_{i}||>C^{k},
$$

where $C$ is a positive constant, one easily verifies

LEMMA 7.

$$
m\left(r, \frac{\eta^{\prime}(f) f^{\prime}}{\eta(f)}\right) \leqq C \log ^{+} k+O(\log r T(r, f))
$$

$C$ a constant, for all $r$ in $S(k)$ except a set $E_{0}$ of finite measure. $E_{0}$ and $O(\log r T(r, f))$ are independent of $k$.

Also one verifies easily

LEMMA 8. $T(r, \eta(f)) \geqq k T(r, f)-k \log 4 A$.

LEMMA 9. $T(r, \eta(f)) \equiv T(r, 1 / \eta(f))+\log k(A B)^{k}$, where $B=\max \left|b_{i}\right|, f(z)=\sum_{i=0}^{\infty} b_{i} z^{i}$.

Proof. $T(r, \eta(f))=T(r, 1 / \eta(f))+\log \left|C_{\lambda}\right|$, where $\eta(f)=C_{\lambda} z^{\lambda}+\cdots, C_{\lambda} \neq 0$. Clearly $\left|C_{\lambda}\right| \leqq k(A B)^{k}$.

As an immediate consequence of the above lemmas we have

THEOREM 11. Suppose $f(z)$ is a nonconstant meromorphic function with $f(0) \neq 0, \infty$. For any sequence $a_{1}, a_{2}, \ldots,\left|a_{i}\right| \leqq\left|a_{i+1}\right|$, with $a_{i} \neq f(0)$, let $\delta(k)=$ minimum of the distances between the first $k$ points of the sequence. Then for ever: $k \geqq 2$,

$$
\begin{aligned}
(k-1) T(r, f) \leqq & N(r, f)+\sum_{i=1}^{k} N\left(r, \frac{1}{f-a_{i}}\right)-\left\{2 N(r, .)+N\left(r, \frac{1}{f^{\prime}}\right)-N\left(r, f^{\prime}\right)\right\} \\
& +C^{\prime} k \log r+4(k-1) \log \frac{1}{\delta(k)}+O(\log (r T(r, f)))
\end{aligned}
$$

for all $r$ in $S(k)$ outside a set ${ }^{r}$, of finite measure. $E_{\text {, }}$ and $O(\log r T(r, f))$ do not depend on $k$. 
REMARK. The above proof is a refinement of an unpublished proof of Nevanlinna's second fundamental theorem given by P. C. Rosenbloom during a series of lectures at Syracuse University a few years ago.

Definition. Let $f(z)$ and $g(z)$ be any two meromorphic functions. We shall say that $f(z)$ grows faster than $g(z)$ almost everywhere, denoted by $G(f)>$ a.e. $G(g)$, if and only if

$$
\lim _{r \rightarrow \infty ; r \in E} T(r, f) / T(r, g)=\infty
$$

where $E$ is the complement of a set of finite measure.

THEOREM 12. Let $f(z)$ and $g(z)$ be any two meromorphic functions and let $S$ and $T$ be any finite sets containing three or more points of the extended plane. If $f(z) \in S$ iff $g(z) \in T$ then $G(f) \ngtr$ a.e. $G(g)$.

Proof. Let $a_{1}, a_{2}$, and $a_{3}$ be distinct elements of $S$ and $b_{i}, i=1, \ldots, k$ be the elements of $T$. We use the definition of $\bar{N}(r, 1 /(f-a))$ with multiple poles being counted only once.

Assume that $G(f)>$ a.e. $G(g)$. Thus we have

We have

$$
\lim _{r \rightarrow \infty ; r \in E} T(r, f) / T(r, g)=\infty
$$

$$
\bar{N}\left(r, \frac{1}{f-a_{i}}\right) \leqq \sum_{j=1}^{k} \bar{N}\left(r, \frac{1}{g-b_{j}}\right)
$$

for each $i=1,2,3$. Thus for any $\varepsilon>0$,

$$
\frac{\bar{N}\left(r, 1 /\left(f-a_{i}\right)\right)}{T(r, f)} \leqq \sum_{j=1}^{k} \frac{\bar{N}\left(r, 1 /\left(g-b_{j}\right)\right)}{T(r, f)}<\varepsilon
$$

for sufficiently large $r$ in $E$. This, however, contradicts Nevanlinna's second fundamental theorem.

THEOREM 13. If $f$ is transcendental meromorphic and of finite order and $g$ is entire of positive lower order, then

$$
T(r, f(g(g))) / T(r, f) \rightarrow \infty
$$

outside a set of finite measure.

Proof. We first note that for any transcendental entire $g$ and $f$ as above

$$
T(r, f(g)) / T(r, g) \rightarrow \infty
$$

as $r \rightarrow \infty$ (see Hayman, p. 54). Though Hayman stated (9) for entire $f$, it remains valid for meromorphic $f$ as well.

It is well known, however, that since $g$ is of positive lower order, $g g$ must be of infinite lower order, i.e. for any constant $C$

$$
\liminf _{r \rightarrow \infty} \frac{\log T(r, g g)}{\log r}>c
$$

or $T(r, g g)>r^{c}$ for sufficiently large $r$. 
Since $f$ is of finite order we also have for some $c^{\prime}<c$ that

$$
T(r, f)<r^{c^{\prime}}
$$

for sufficiently large $r$.

Thus

$$
T(r, g g) / T(r, f)>r^{c-c^{\prime}} \rightarrow \infty
$$

with $r$. Thus

$$
T(r, f(g(g))) / T(r, f) \rightarrow \infty
$$

with $r \rightarrow \infty$.

THEOREM 14. If $f(z)$ is any transcendental entire function and $S$ is any finite set containing three or more elements, then $f^{-1}(S)$ is not a reduced set of any entire function $g(z)$.

Proof. Assume that $f^{-1}(S)$ is a reduced set of $g(z)$. Then $g\left(f^{-1}(S)\right)=f^{-1}(S)$ and $f\left(g\left(f^{-1}(S)\right)\right)=f\left(f^{-1}(S)\right)=S$. On the other hand, if $f(g(x))=s \in S$, then $g(x) \in f^{-1}(S)$ and hence $x \in f^{-1}(S)$. Hence $f(g(z)) \in S$ if and only if $f(z) \in S$ and by virtue of Theorem $12 G(f(g)) \ngtr$ a.e. $G(f)$. But by Theorem 13, $G(f(g))>$ a.e. $G(f)$ so that our theorem follows.

In a similar manner one proves

THEOREM 15. If $f(z)$ is transcendental and meromorphic of finite order and $S$ is any finite set with 3 or more elements, then $f^{-1}(S)$ is not a reduced set of any entire function $\mathrm{g}(\mathrm{z})$ of positive lower order.

The argument is the same as before once we note that $S$ is a reduced set of $g g$ whenever it is a reduced set of $g$.

NoTE. We have actually proved more than is stated in Theorems 14 and 15 since no assumption need be made about the multiplicity of the roots of $g(z)-t$, where $t \in f^{-1}(S)$.

COROLLARY 1. The set of rational integers is not a reduced set of any transcendental entire functions.

COROLlaRY 2. A point lattice is not a reduced set of any transcendental entire function.

Proof. One can easily construct a Weierstrass $p$-function $f$ which maps a given point lattice into a finite set $\left\{a_{1}, a_{2}, a_{3}, \ldots\right\}$ having more than two points such that no other complex numbers go into these points.

THEOREM 16. Let $S=\left\{a_{i}\right\}, i=1,2, \ldots$, be a discrete set, $\left|a_{i}\right|<\left|a_{i+1}\right|$. If $S$ is a reduced set of a transcendental entire function $f$, then for every $t, \delta\left(n_{f}(r, S)\right) \leqq r^{-t}$ for all $r$ outside a set of finite measure. If in addition $f$ is of lower order $<1 / 2$, then $S$ cannot be a reduced set of $f$. Here $n_{f}(r, S)$ is the number of elements of $S$ in $|z| \leqq r$ 
and for any positive integer $k, \delta(k)=$ minimum of the distances between the first $k$ elements of the sequence $S$.

Proof. First assume that $f$ has positive lower order. Then $g=f \circ f$ has infinite lower order and $S$ is also a reduced set for $g$. Let $a_{1}, a_{2} \in S\left(a_{1}, a_{2}\right.$ finite), then it follows from the standard form of the Second Fundamental Theorem that

$$
\lim \inf \frac{N\left(r, 1 /\left(g-a_{1}\right)\right)+N\left(r, 1 /\left(g-a_{2}\right)\right)}{r^{\lambda}}=+\infty
$$

for every finite $\lambda$, provided $r \notin E_{0}, E_{0}$ of finite measure. Since $N(r, 1 /(g-a))$ $\leqq n(r, 1 /(g-a)) \log r$, it is immediate that for every finite $\lambda$

$$
\lim \inf \frac{n\left(r, 1 /\left(g-a_{1}\right)\right)+n\left(r, 1 /\left(g-a_{2}\right)\right)}{r^{\lambda}}=+\infty \quad\left(r \notin E_{0}\right) .
$$

Hence for any real $\lambda$ and all $r$ sufficiently large $\left(r \notin E_{0}\right), n(r, S) \geqq r^{\lambda}$ (since $\left.g^{-1}\left(\left\{a_{1}, a_{2}\right\}\right) S\right)$. If $\delta(n(r, S))>1 / r^{\lambda-1}$ for some $\lambda$ and $r$; then $n(r, S) \leqq r / \delta(n(r, S))<r^{\lambda}$. In view of the preceding, this is only possible if $r<r_{0}(\lambda)$ for a suitable $r_{0}$ or if $r \in E_{0}$.

For $f$ of lower order $<1 / 2$, denoting by $\mu(r, f)$ the minimum modulus of $f$ on $|z|=r$, Kjellberg [2] has extended Wiman's Theorem and has shown that

$$
\limsup _{r \rightarrow \infty}\{\log \mu(r, f) / \log M(r, f)\} \geqq c>0,
$$

where $c$ depends only on the lower order. Let $\left\{r_{k}\right\}$ be an increasing unbounded sequence for which this inequality holds. Since $f$ transcendental implies that $\lim \inf _{r \rightarrow \infty}\{\log M(r, f) / \log r\}=+\infty$; choosing any $\lambda>1$, there exists $k_{0}$ so that for $k>k_{0}, \mu\left(r_{k}, f\right) \geqq r_{k}^{\lambda}$. Let $S$ consist of the sequence $\left\{c_{i}\right\}$ and denote by $D_{r}$ the open disc centered at the origin of radius $r$. Since a nonconstant entire function of lower order $<1 / 2$ has no finite Picard exceptional value, for all sufficiently large $k>k_{1}$, $0 \in f\left(D_{r_{k}}\right)$. But $\mu\left(r_{k}, f\right) \geqq r_{k}^{\lambda}$ for $k>k_{0}$. Hence for $k>\max \left(k_{0}, k_{1}\right)$, we must have $D_{r_{k}^{\lambda}} \subset f\left(D_{r_{k}}\right)$ and in particular $D_{r_{k}} \subset f\left(D_{r_{k}}\right)$. Since $S$ is a reduced set for $f$ each $c_{i}$ with $\left|c_{i}\right|<r_{k}$ must have a pre-image among the $c_{i}$ 's in $D_{r_{k}}$, i.e. $f^{-1}\left(c_{i}\right)=c_{j} \in D_{r_{k}}$. Hence each $c_{i} \in D_{r_{k}}$ has one and only one pre-image in $D_{r_{k}}$. Keeping $c_{i}$ fixed and letting $k \rightarrow \infty$, we see that $c_{i}$ has exactly one pre-image in the plane. Thus $f$ must be a linear polynomial by Picard's Theorem.

It is worth noting that if $S$ is a discrete infinite set, then $S$ cannot be a reduced set of a meromorphic function which is not entire.

This clearly gives us another proof of the fact that a point lattice is not a reduced set of any transcendental entire function.

If $f$ is transcendental entire and $S$ is a finite set with two or more elements, say $\left\{a_{1}, a_{2}, \ldots\right\}$ then it follows from the standard form of Nevanlinna's second fundamental theorem that for every $\varepsilon>0$

$$
n\left(r, f^{-1}(S)\right) \geqq(1-\varepsilon)(n(r, S)-2) T(r, f) / \log r
$$


one can easily verify that (10) remains valid if $f$ is transcendental meromorphic and not entire and $S$ is an infinite discrete set.

Actually more is true.

THEOREM 17. Let $f(z)$ be a transcendental meromorphic function $f(0) \neq 0, \infty$. If a discrete set $S=\left\{a_{1}, a_{2}, \ldots\right\}, a_{i} \neq f(0)$, with three or more elements, satisfies for some $t$,

$$
\delta(n(r, S)) \geqq r^{-t}
$$

for a set, $E$, of $r$ of infinite measure, then for every $\varepsilon>0$

$$
n\left(r, f^{-1}(S)\right) \geqq(1-\varepsilon)(n(r, S)-2) T(r, f) / \log r
$$

for all $r$ in $E$ outside a set of finite measure. Here $n(r, S)$ denotes the number of elements of $S$ in $|z| \leqq r$.

Proof. One easily verifies

$$
\sum_{i=1}^{n(r, S)} \bar{N}\left(r, f, a_{i}\right) \leqq \sum_{j=1}^{n(r, f-1(S))} \log \frac{r}{\left|b_{j}\right|}+A \log r
$$

where $b_{j}$ are the totality of $a_{i}$-points of $f(i=1,2, \ldots)$.

Thus

$$
(n(r, S)-2) T(r, f) \leqq n\left(r, f^{-1}(S)\right)\left(\log r+A^{\prime}\right)+C n(r, s) \log r+O(\log r T(r, f))
$$

for all $r$ in $E$ outside of finite measure; $C, A^{\prime}$ constant. Our theorem follows.

COROLlARY 1. If $f(z)$ and $S$ are as in Theorem 17, then for any constant $k$ $n\left(r, f^{-1}(S)\right)>k n(r, S)$ for all $r$ in $E$ outside a set of finite measure.

COROLlaRY 2. If $f(z)$ is a transcendental meromorphic function and $S$ is any lattice, then $f^{-1}(S)$ is not a lattice.

Proof. Choose $A$ such that $f(A) \neq 0, \infty, a_{i}, i=1,2, \ldots$, where $S=\left\{a_{1}, a_{2}, \ldots\right\}$. $f(z+A)$ satisfies the hypotheses of the above corollary and our assertion follows.

COROLlaRY 3. Let $a_{i}, i=1,2,3$ be any three numbers in the extended complex plane. For any positive real numbers $n$ and $m$ let

$$
S_{n m}=\left\{A^{n}+i B^{m} ; A \text { and } B \text { any integers }\right\} .
$$

If $f(z)$ is a meromorphic function such that its only $a_{i}$-points for $i=1,2,3$ are in $S_{n m}$, then $f(z)$ is at most of lower order $1 / n+1 / m$.

COROLLARY 4. If for any meromorphic function, $f$, there exist $a_{i} ; i=1,2,3$, satisfying the hypotheses of Corollary 2 and a point a such that $f^{-1}(a) \in S_{n^{\prime} m^{\prime}}, n^{\prime}, m^{\prime}$ positive reals, then

$$
1 / n^{\prime}+1 / m^{\prime} \leqq \rho_{L} \leqq 1 / n+1 / m,
$$

where $\rho_{L}$ denotes the lower order of $f$. In particular when $n=n^{\prime}$ and $m=m^{\prime}, f$ must be of lower order $1 / n+1 / m$. 
We note that for $n=1$ and $m=1$ this corollary is sharp as illustrated by the Weierstrass $p$-function.

As an extension of Theorem 16 we state

THEOREM 18. A finite set is not a reduced set of any rational function other than a linear transformation.

The proof is trivial.

THEOREM 19. Let $f(z)$ be a meromorphic function and let $S$ be any set containing three or more points. If the infimum of the distances between points of $f^{-1}(S)$ is nonzero then $f(z)$ is of lower order less than or equal to 2.

Proof. We may assume that $f$ and $S$ satisfy the hypotheses of Theorem 17 . The theorem follows by noting that $n\left(r, f^{-1}(S)\right) \leqq C r^{2} ; C$ a constant.

The Weierstrass $p$-function illustrates the sharpness of this theorem also.

As we have indicated in Corollary 4, one can also get lower bounds for the lower order of meromorphic functions, provided that the poles of a point are at least of a certain density. Much more is known about lower bounds for order and lower order in the case of entire functions (see [4], [3]). One could easily state theorems of the same type as Corollary 4, which satisfy somewhat different conditions. As an illustration we prove

THEOREM 20. If for any entire function, $f$, there exist $a_{i}, i=1,2,3$, satisfying the hypotheses of Corollary 3, with $n$ and $m$ greater than 2 and if for some number $a$ $f^{(k)}(a)$ is a Gaussian integer for sufficiently large $k$, then $f$ is a polynomial.

Proof. One need only note that by the first hypothesis $f$ is at most of lower order less than 1 , while by the second hypothesis $f$ must be at least of lower order 1 unless it is a polynomial.

A number of questions remain open. Do there exist discrete sets $S$ containing three or more elements which are reduced sets of a transcendental meromorphic function? Can nonlinear polynomials have discrete reduced sets?

One could also ask questions about nondiscrete denumerable sets. However, the methods of this paper could not be used to deal with such problems.

REMARK. For any entire function $f$, the sets $S$ formed by taking all combinations of images and pre-images of a denumerable set, $T$, i.e. $f_{n} f_{m}^{-1} \cdots f_{t}(T)$ certainly satisfies $f^{-1}(S)=S$. It is difficult to determine, however, whether or not $S$ is discrete. E. G. Straus suggested that $S$ is even dense.

The author is indebted to the referee for useful remarks and for the statement and proof of the second half of Theorem 16.

\section{REFERENCES}

1. W. K. Hayman, Meromorphic functions, Oxford Mathematical Monographs, Clarendon Press, Oxford, 1964.

2. B. Kjellberg, On the minimum modulus of entire functions, Math. Scand. 8 (1960), 189-197. 
3. A. L. Rubel and S. Hellerstein, Subfields that are algebraically closed in the fields of all meromorphic functions, Technical Rep. No. 3, Appl. Math. and Statist. Labs., Stanford Univ., Calif., 1963.

4. A. Selberg, Uber ganzwertige ganze tranzendente Funktionen. I, Arch. Math. Natur. 44 (1941), 45-52.

5. E. G. Straus, On entire functions with algebraic derivatives at certain algebraic points, Ann. of Math. 52 (1950), 188-198.

6. E. C. Titchmarsh, The theory of functions, 2nd ed., Oxford Univ. Press, Oxford, 1939.

7. R. Nevanlinna, Le théorème de Picard-Borel et la théorie des functions méromorphes, Gauthier-Villars, Paris, 1929.

U. S. Naval Research Laboratory, WASHINGTON, D. C. 